\title{
Luxação distal isolada do pisiforme: Relato de caso*
}

\section{Isolated distal pisiform dislocation: Case case}

\author{
Rafael Dias ${ }^{1}$ [ Teresa Alves-da-Silva $^{1}$ Carlos Martinho ${ }^{1}$ Francisco Guerra-Pinto ${ }^{1}$ \\ ${ }^{1}$ Departamento de Especialidades Cirúrgicas, Unidade Funcional de \\ Ortopedia, Hospital de Cascais - Dr. José de Almeida, Alcabideche, \\ Portugal \\ Endereço para correspondência Rafael Dias, MD, Avenida Brigadeiro \\ Novais, 2755-099, Alcabideche, Portugal \\ (e-mail: rafaelrmdias@gmail.com).
}

Rev Bras Ortop 2022;57(2):341-344.

\section{Resumo \\ Palavras-chave \\ - traumatismos do punho \\ - ossos do carpo \\ - luxações articulares \\ - pisiforme}

\section{Abstract}

\section{Keywords}

- wrist injuries

- carpal bones

- joint dislocations

- pisiform
A luxação isolada do pisiforme é uma lesão rara com poucos casos descritos na literatura. Esse tipo de lesão é observado tipicamente em adultos jovens do sexo masculino e pode ser facilmente negligenciada numa primeira avaliação. A luxação proximal isolada é mais comum devido à ação do flexor ulnar do carpo (FUC).

Apresentamos o caso de um paciente do sexo masculino, com 19 anos de idade, com luxação distal isolada do pisiforme após traumatismo do punho. O paciente foi submetido a uma redução aberta e fixação interna com fios de Kirschner com excelente resultado funcional.

Apesar de não existir um método de tratamento consensual, a redução fechada perfila-se como tratamento de primeira linha na apresentação aguda. Em caso de insucesso ou como método alternativo pode-se optar pela redução aberta ou a excisão do pisiforme.

Isolated pisiform dislocation is a rare lesion with few cases described in the literature. This type of lesion is typically observed in young males and can be easily overlooked in the first assessment. Isolated proximal dislocation is more common due to the action of the flexor carpi ulnaris (FCU) muscle.

We present the case of a 19-year-old male patient with isolated distal pisiform dislocation after wrist trauma. He underwent open reduction and internal fixation with Kirschner wires with excellent functional outcomes.

Although there is no consensual therapeutic method, closed reduction is a first-line treatment for acute presentations. Pisiform open reduction or excision may be performed alternatively or after a failed closed reduction.
Trabalho desenvolvido no Departamento de Especialidades Cirúrgicas - Unidade Funcional de Ortopedia, Hospital de Cascais Dr. José de Almeida, Alcabideche, Portugal. recebido

06 de Junho de 2020

aceito

17 de Setembro de 2020

Publicado on-line

Março 31, 2021
DOI https://doi.org/

10.1055/s-0040-1722589.

ISSN 0102-3616. (c) 2021. Sociedade Brasileira de Ortopedia e Traumatologia. All rights reserved.

This is an open access article published by Thieme under the terms of the Creative Commons Attribution-NonDerivative-NonCommercial-License, permitting copying and reproduction so long as the original work is given appropriate credit. Contents may not be used for commercial purposes, or adapted, remixed, transformed or built upon. (https://creativecommons.org/ licenses/by-nc-nd/4.0/)

Thieme Revinter Publicações Ltda., Rua do Matoso 170, Rio de Janeiro, RJ, CEP 20270-135, Brazil 


\section{Introdução}

A luxação do pisiforme é uma patologia rara contando com menos de 30 casos documentados. ${ }^{1-7}$ A sua localização dentro do tendão do flexor ulnar do carpo (FUC) conferelhe estabilidade intrínseca, tornando a luxação pouco provável, especialmente sem outras lesões associadas. Com escassos casos descritos na literatura, a abordagem terapêutica ideal ainda não é clara.

\section{Caso clínico}

Apresentamos um paciente do sexo masculino, com 19 anos de idade, com traumatismo da face volar do punho esquerdo durante elevação de um objeto pesado (mobília). A compressão contínua infligida pelo peso do objeto no punho em extensão culminou com o início súbito de dor e discreta incapacidade funcional do punho. Na admissão ao serviço de urgência, o paciente apresentava dor e edema do bordo ulnar do punho e mão esquerdos. Referia discreta limitação da flexão do punho e não mostrava sinais de compromisso neurológico.

A radiografia revelou luxação distal do pisiforme até ao nível do hâmulo do hamato (-Figura 1). A tomografia computorizada não mostrou quaisquer outras lesões associadas (-Figura 2). Foi realizada uma tentativa de redução fechada sem sucesso. Assim, o punho foi imobilizado com tala gessada posterior antebraqui-palmar e foi proposta a intervenção cirúrgica.

Foi realizada redução aberta do pisiforme e fixação ao piramidal com dois fios de Kirschner, sob controle radio-

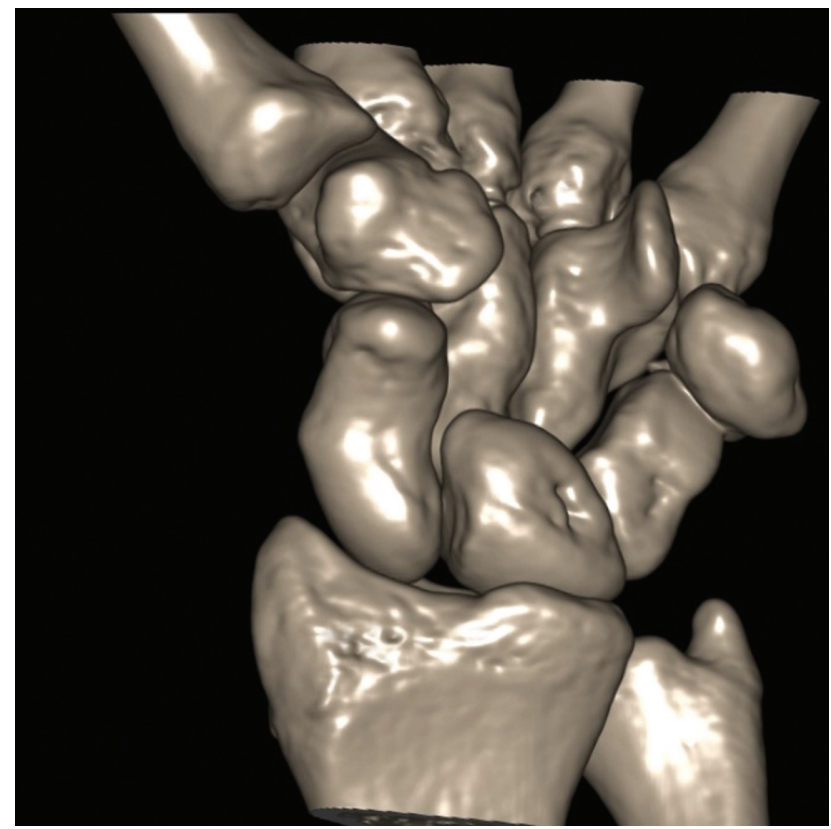

Fig. 2 Tomografia computorizada (reconstrução tridimensional) a demostrar a luxação do pisiforme sem outras lesões associadas.

scópico (-Figura 3). Constatou-se disrupção da cápsula articular e integridade do FUC mantida. O punho foi imobilizado com tala gessada posterior antebraqui-palmar em flexão e desvio ulnar.

Cinco semanas após a cirurgia, foram removidos os fios de Kirschner e a tala gessada, e foi iniciada a reabilitação motora.

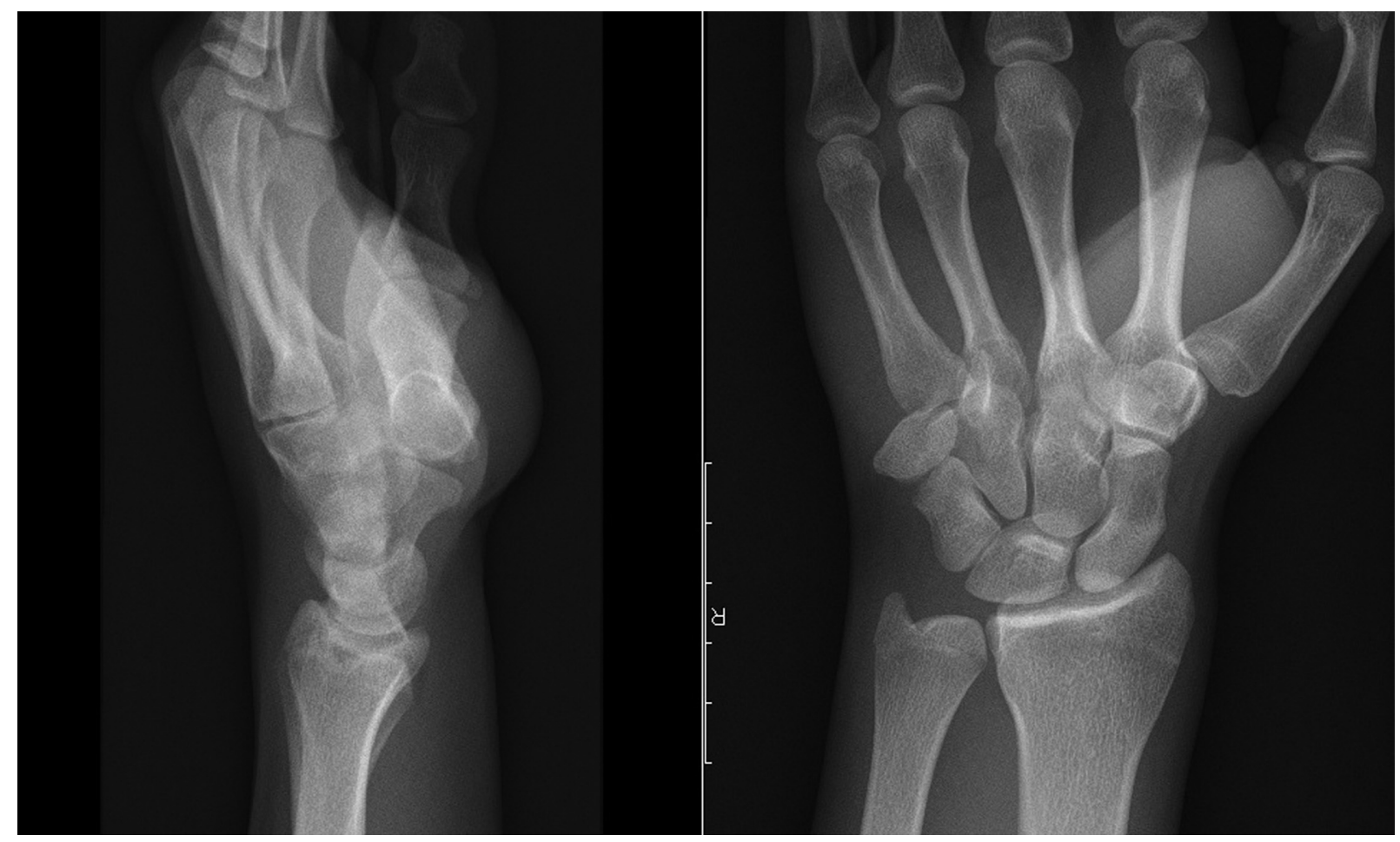

Fig. 1 Radiografia inicial a demonstrar a luxação distal do pisiforme. 


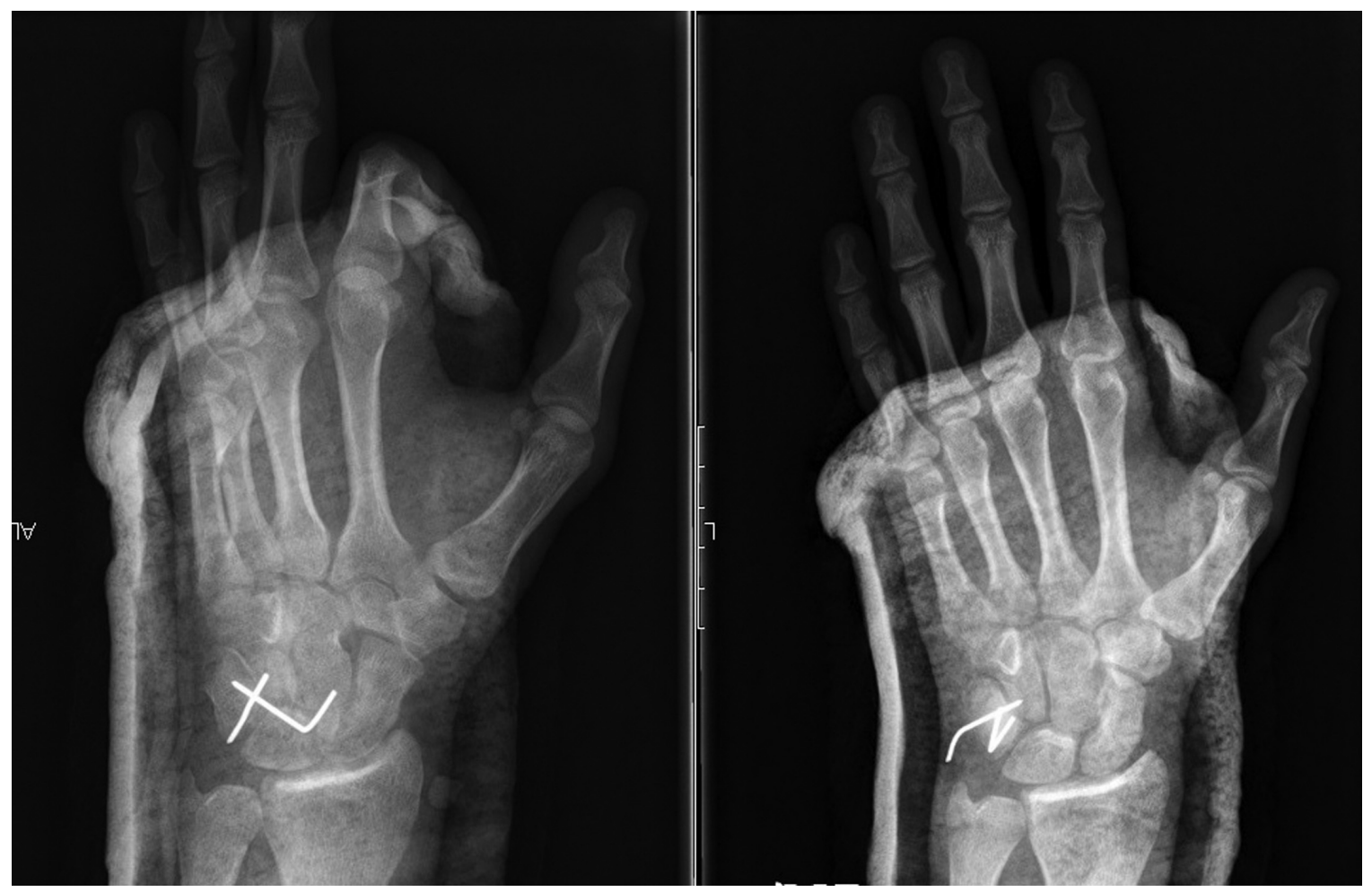

Fig. 3 Radiografia no período pós-operatório imediato a demonstrar a redução anatómica do pisiforme, fixado ao piramidal com fios de Kirschner.
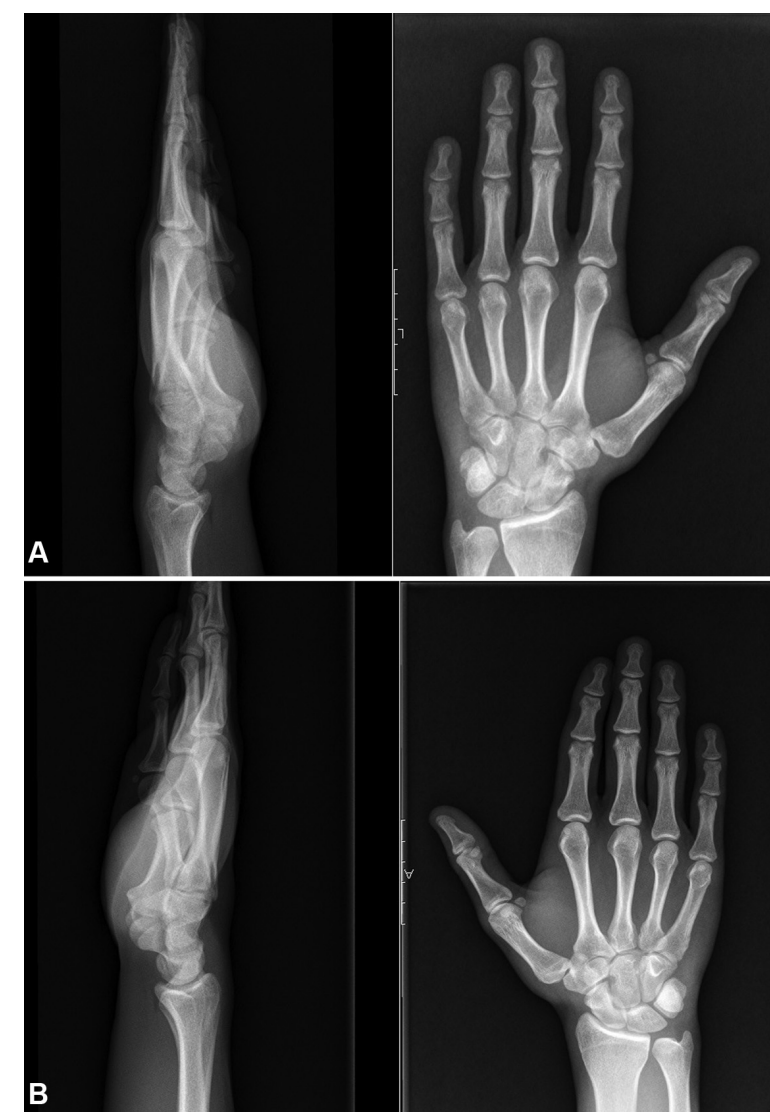

Fig. 4 (A) Radiografia do punho esquerdo às 12 semanas pósoperatório: redução do pisiforme mantida; (B) Radiografia do punho direito.
Às 12 semanas de pós-operatório, a radiografia de controle mostrava o pisiforme na sua posição nativa (- Figura 4). Aos 12 meses de pós-operatório, o paciente apresentava recuperação completa do arco de movimento do punho, e força muscular simétrica sem dor residual ( - Figura 5) - Disability of Arm, Shoulder and Hand (DASH) escore 0; Patient-Rated Wrist Evaluation (PRWE) escore 0.

\section{Discussão}

A luxação isolada do pisiforme é uma lesão extremamente rara, com um número reduzido de casos descritos na literatura. É uma lesão que pode facilmente ser negligenciada, especialmente quando associada a outras lesões. Assim, é necessário um alto grau de suspeição para identificar esta lesão em pacientes com traumatismo do membro superior.

Situado no bordo ulnar da fileira proximal do carpo, o osso pisiforme articula com o piramidal através da sua face dorsal. Em razão da sua faceta articular plana, o pisiforme depende dos tecidos moles envolventes para garantir a sua estabilidade, como os ligamentos pisohamato e pisometacárpico e os tendões do FUC e do abdutor do quinto dedo. $\mathrm{O}$ pisiforme atua como osso sesamoide, servindo de alavanca para aumentar a força de flexão do punho produzida pelo FUC. ${ }^{1,8,9}$

Existem dois mecanismos propostos para explicar a luxação do pisiforme: traumatismo externo direto ou força tracional excessiva exercida pelo FUC. $^{2-4} \mathrm{O}$ último mecanismo parece ser o mais frequente, quer por queda com 


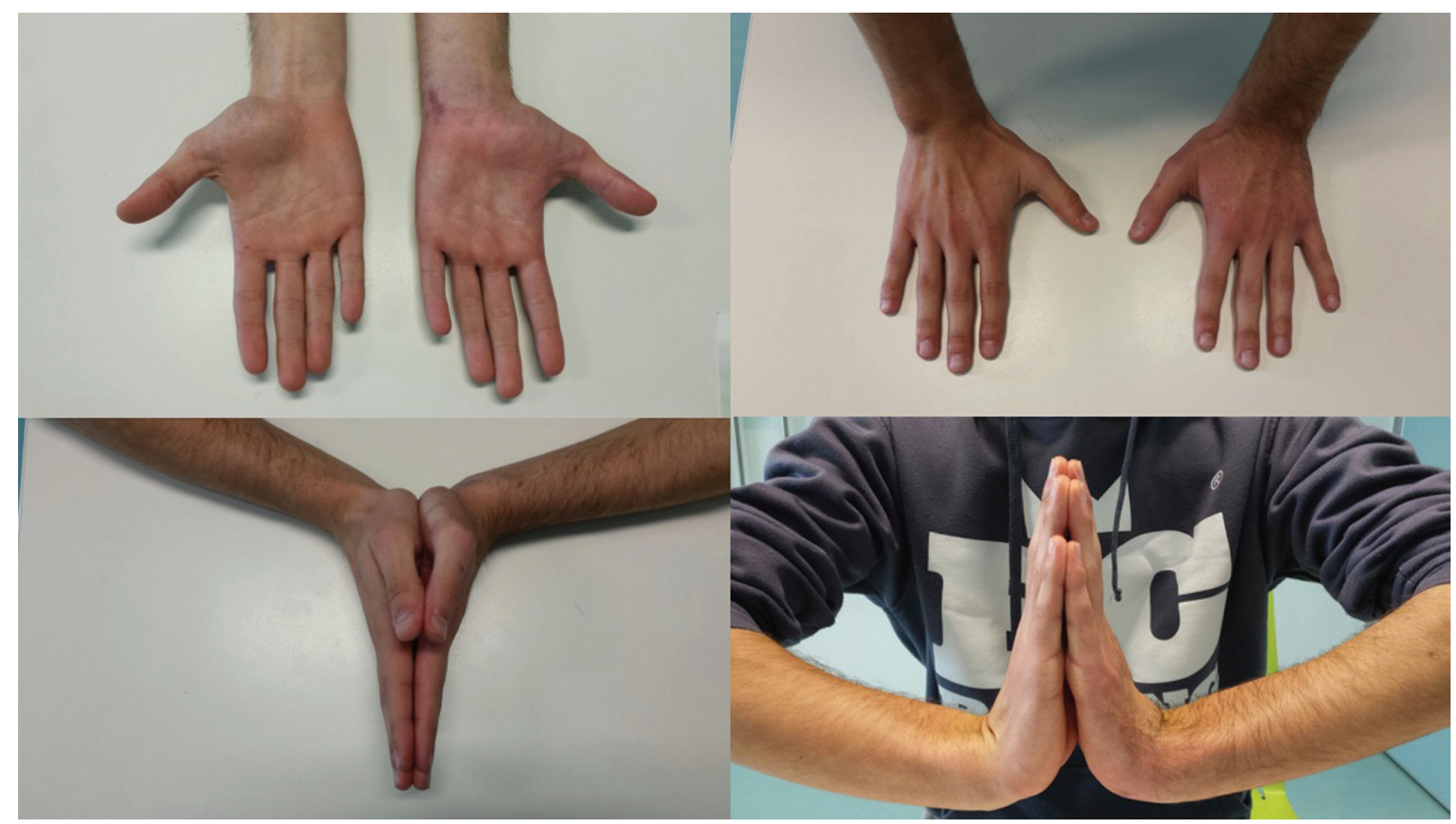

Fig. 5 Fotografia do paciente a demonstrar um arco de movimento simétrico.

traumatismo do punho em extensão, quer por contração muscular violenta súbita do FUC.

A ação do músculo flexor do punho predominante - FUC tende a deslocar o pisiforme proximalmente. Assim, a luxação do pisiforme neste sentido é a mais frequente devido à sobreposição das forças do FUC às demais estruturas estabilizadoras. $^{5}$

No caso descrito, o mecanismo traumático específico pode explicar a fisiopatologia da luxação do pisiforme no sentido distal. Hipotetizamos que a força tangencial progressiva exercida pelo objeto pesado sobre o punho pode explicar a migração distal do pisiforme, devido à disrupção capsular e ao estiramento do tendão do FUC. O hâmulo do hamato pode atuar como fator de bloqueio à redução do pisiforme para a sua posição anatômica. A força exercida pelo abdutor do quinto dedo pode representar outro cofator da migração do pisiforme para a fileira distal do carpo.

O tratamento indicado ainda não é claro; no entanto, uma tentativa de redução fechada num traumatismo agudo pode ser a primeira opção terapêutica. Caso a redução fechada se mostre infrutífera, deve ser realizada a redução aberta e fixação interna ou a excisão do pisiforme. Nos casos de tratamento não imediato ou atraso diagnóstico, a redução anatómica pode tornar-se difícil e a excisão do pisiforme apresenta-se como o tratamento com resultados mais previsíveis. ${ }^{3,6}$

No caso descrito, optou-se pela redução aberta e fixação do pisiforme na sua posição anatômica, tendo em conta a possibilidade de recuperação total da função do FUC. A imobilização em flexão e desvio ulnar permite diminuir a tensão sobre o FUC para potenciar a sua cicatrização, bem como a das outras estruturas justa-articulares. ${ }^{8}$ Acreditamos que a técnica utilizada é um método fiável e com bom resultado funcional para o tratamento da luxação isolada do pisiforme.

\section{Conflito de Interesses}

Os autores declaram não haver conflito de interesses. O estudo foi realizado em conformidade com a Declaração da Associação Médica Mundial de Helsinque sobre Princípios Éticos para Pesquisa Médica Envolvendo Seres Humanos.

\section{Referências}

1 Kwon OS, Choi SP, Won HY. Acute isolated pisiform dislocation: A case report. J Korean Orthop Assoc 2007;42(05):688-691

2 Minami M, Yamazaki J, Ishii S. Isolated dislocation of the pisiform: a case report and review of the literature. J Hand Surg Am 1984;9A (01):125-127

3 Schädel-Höpfner M, Junge A, Böhringer G. [Dislocation of the pisiform bone. A review of the literature]. Handchir Mikrochir Plast Chir 2002;34(03):168-172

4 Sharara KH, Farrar M. Isolated dislocation of the pisiform bone. J Hand Surg [Br] 1993;18(02):195-196

5 Campbell E, Magi E. A dislocated pisiform: Case report. Can J Plast Surg 1999;7(02):57-58

6 Goriainov V, Bayne G, Warwick DJ. Traumatic dislocation of the pisiform: a case report. J Orthop Surg (Hong Kong) 2010;18(03): 389-390

7 Kubiak R, Slongo T, Tschäppeler $\mathrm{H}$. Isolated dislocation of the pisiform: an unusual injury during a cartwheel maneuver. J Trauma 2001;51(04):788-789

8 Pevny T, Rayan GM, Egle D. Ligamentous and tendinous support of the pisiform, anatomic and biomechanical study. J Hand Surg Am 1995;20(02):299-304

9 Moojen TM, Snel JG, Ritt MJ, Venema HW, den Heeten GJ, Bos KE. Pisiform kinematics in vivo. J Hand Surg Am 2001;26(05): 901-907 